\title{
RESEARCH
}

\section{Reflective to be Effective: Establishing Validity Evidence for Parallel Patient-Provider Empathy Scales to Drive Self-Reflection}

\author{
Michelle L. Blakely, PhD, MEd, ${ }^{\mathrm{a}, \mathrm{b}}$ Benjamin D Aronson, PharmD, PhD, ${ }^{\mathrm{c}}$ Aleda M. H. Chen, PharmD, PhD, ${ }^{\mathrm{d}}$ \\ Mary E Kiersma, PharmD, PhD, ${ }^{\mathrm{e}}$ Emily Wicker, PharmD, ${ }^{\mathrm{d} J u a n i t a} \mathrm{~A}$. Draime, PharmD ${ }^{\mathrm{d}}$ \\ ${ }^{a}$ University of Wyoming, School of Pharmacy, Laramie, Wyoming \\ ${ }^{\mathrm{b}}$ Editorial Board Member, American Journal of Pharmaceutical Education, Arlington, Virginia \\ ${ }^{c}$ Ohio Northern University, Raabe College of Pharmacy, Ada, Ohio \\ ${ }^{d}$ Cedarville University, School of Pharmacy, Cedarville, Ohio \\ ${ }^{\mathrm{e}}$ Accreditation Council for Pharmacy Education (ACPE), Chicago, Illinois
}

Corresponding Author: Aleda M. H. Chen, Cedarville University, School of Pharmacy, 251 N. Main St., Cedarville, OH 45314. Tel: 937-766-7454. Email: amchen@cedarville.edu

Submitted July 7, 2021; accepted November 12, 2021; ePublished December 2021

Objective. Student pharmacists must cultivate self-awareness to ensure that they can assess their skill development and abilities, including affective domain skills such as empathy. External feedback can augment development, but validated assessments are needed for accuracy. Thus, the objectives of this study were to: establish validity evidence of the KCES-R (Kiersma-Chen Empathy Scale-Revised), compare student self-perceptions and standardized patient (SP) perceptions of student empathy using a parallel patient scale (KCES-PV), and evaluate student reflections on the encounter.

Methods. Student pharmacists completed an assessment of their self-perceptions of empathy (KCES-R) pre-/post-patient encounter. SPs completed the KCES-PV regarding student pharmacist empathy immediately post-encounter. Student pharmacists also watched their encounter videos and completed a self-reflection on their use of empathy. Descriptive statistics, Spearman's rank-order correlations, exploratory factor analysis, Cronbach's alpha, and Wilcoxon Signed-Rank test were computed. Reflections were explored using thematic analysis.

Results. The KCES-R contains 2 factors with high internal consistency and shows the ability to detect changes in empathy. Student pharmacist self-perceptions of empathy ability appeared higher than the SP evaluation. Student pharmacists had a stronger belief in the importance of expressing empathy during patient encounters and indicated a need for further development.

Conclusion. This study provides validity evidence for the use of the KCES-R and presents a parallel scale that may be used by SPs. Validated parallel scales along with reflective practice could be a potential avenue to grow self-awareness and empathy by allowing students to receive feedback and then reflect on their perceived vs actual demonstration of the skill.

Keywords: Kiersma Chen Empathy Scale (KCES); empathy; pharmacy; validity evidence; self-assessment

\section{INTRODUCTION}

Empathy is the ability to understand someone's situation, communicate that understanding, and then act upon it. ${ }^{1}$ Empathy is an important aspect of health care and has been shown to improve patient outcomes. ${ }^{1}$ Empathy includes cognitive and affective elements, is multi-dimensional - including behavior, feelings, attitudes, and knowledge - and allows health care professionals to challenge assumptions and build relationships. ${ }^{1}$ This is a key component of providing culturally sensitive care, and empathetic individuals have effective cross-cultural communication. ${ }^{1}$ Through empathy, health care professionals can increase trust, patient adherence, and health outcomes. ${ }^{1}$ The Accreditation Council for Pharmacy Education (ACPE) 2016 Standards list empathy as a communication skill that student pharmacists should develop before Advanced Pharmacy Practice Experiences (APPEs). ${ }^{2}$ The Center for the Advancement of Pharmacy Education's (CAPE) Educational Outcomes also include empathy as an example of domain 3.6: "Effectively communicate verbally and nonverbally when interacting with an individual, group, or organization.",2,3

Empathy can be challenging to assess. Self-assessment of empathy is a typical approach, and an important ability for student pharmacists to develop. CAPE domain 4.1 focuses on student pharmacists' ability to judge their own personal and professional skills and abilities. ${ }^{3}$ However, self-assessments are limited by self-biases which commonly lead to overestimation of personal abilities (ie, knowledge, leadership skills, social skills). ${ }^{4}$ In order to help address 
overestimation of ability, student pharmacists should also receive external feedback in order to improve their awareness and ability to self-assess. ${ }^{3}$ While teachers and peers can provide valuable external feedback, patient assessment of empathy could help student pharmacists and other health care providers to better understand how patients perceive them and their true ability to empathize. For student pharmacists, Objective Structured Clinical Examinations (OSCEs) are simulated patient encounters with standardized patients (SPs) that allow students to practice and be assessed on their professional skills including empathy. ${ }^{5}$ OSCEs are an opportunity for student pharmacists to self-reflect on the encounter while also receiving feedback from patients and observers. ${ }^{6}$

Affective domain skills can be challenging to accurately evaluate due to their subjective nature and lack of a reference point; thus, measures are needed to accurately assess student pharmacists' skills when interacting with patients. Empathy can be measured with the Kiersma-Chen Empathy Scale (KCES), a validated survey instrument ${ }^{7}$ that has recently been revised. ${ }^{8}$ Other validated tools of empathy exist, such as the Jefferson Scale of Empathy, which was developed through a Delphi process with physicians and validated in other health professions. ${ }^{7}$ The KCES, alternatively, was developed from a definition of empathy and validated in pharmacy and nursing students. ${ }^{7}$

The objectives of this study were to: (1) establish additional validity evidence of the KCES-R (revised version), (2) compare student pharmacists' self-assessment of empathy ability and patient perceptions of student empathy ability using a parallel KCES-PV (patient version), and (3) evaluate student self-assessments of their interaction with a simulated patient.

\section{METHODS}

IRB approval was obtained from Cedarville University and the University of Wyoming. Second-year student pharmacists at Cedarville University School of Pharmacy (CUSOP) and the University of Wyoming (UW) completed OSCEs in the Fall of 2019, and all were invited to participate in the study. Before the OSCE, student pharmacists completed the KCES-R. Then, student pharmacists interacted with the SPs for ten minutes based on a case prompt. CUSOP SPs who are trained in basic communication and role-playing were hired from a local school of medicine's simulation program and received an hour of specific training on each OSCE case. SPs at UW were drama/theater students who were trained by one of the investigators in basic communication, reading patient scripts, and role-playing for one hour prior to interacting with the student pharmacists. The OSCE case used in this study was a homeless patient at a community pharmacy picking-up a new prescription for levetiracetam following an emergency room (ER) visit for a seizure. Student pharmacists were expected to obtain a focused and relevant history, deal with a communication issue, provide patient education, counsel the patient, discuss monitoring plans, and indicate how follow-up should occur. During the OSCE, student pharmacists had the opportunity to show empathy for the patients' concerns over taking a new medication, access to health care, and housing situation. Following the OSCE, student pharmacists completed the KCES$\mathrm{R}$ again and the SPs completed the KCES-PV based upon the specific student encounter. Student pharmacists then watched a recording of their OSCE and completed a written reflection discussing their empathy self-awareness (Figure 1). The prompt for the self-reflection was as follows, "Please describe what you learned about empathy from this activity. Also, what did you learn about self-awareness from this activity? What steps will you take to improve?" There were no word limits or other instructions given regarding the reflection.

The creation and validation of the original KCES has been described previously. ${ }^{7}$ A revised version of the KCES was created using psychometric data from studies that utilized the KCES and cognitive interviews of pharmacy and nursing students. ${ }^{8}$ The KCES-R contains 14 items divided into two sections. The first 7 items assess globally how necessary it is for health care professionals to be able to perform different aspects of empathy (eg, identify with a patient's feelings), with 7 response categories anchored by 'Unnecessary' (1), 'Moderately necessary' (4), and 'Extremely necessary' (7). The second 7 items serve as a self-assessment of personal ability to perform those same aspects of empathy, with 7 response categories anchored by 'Does not describe me' (1), 'Describes me moderately well' (4), and 'Describes me extremely well' (7). Responses were coded as 1 through 7, and scores for each domain were computed by summing items from the domain. ${ }^{8}$ Permission to use the KCES scales at no cost can be obtained by contacting the corresponding author (AC).

Following the creation of the KCES-R, the KCES-PV was developed to parallel the KCES-R with the goal of providing students with external feedback of their empathy, and for comparing student pharmacist self-assessment of empathy ability with patient or SP assessment of student empathy. The KCES-PV has similar questions and response categories as the KCES-R for the first section assessing global views on health care providers being able to perform different aspects of empathy. The second section of the KCES-PV section parallels the KCES-R, but asks about the ability of their health care professional, or student health care professional. The questions are framed from the patient perspective, (eg, identify with my feelings), with seven response options anchored by 'Does not describe them well' (1), 
'Describes them moderately well' (4), and 'Describes them extremely well' (7). Responses were coded as 1 through 7 , and scores for each domain were computed by summing items from the domain.

Data were analyzed using SPSS v 27 (Armonk, NY, USA). First, descriptive (ie, means, standard deviations, median, range, interquartile ranges, skewness, kurtosis, and Shapiro-Wilk statistic) and bivariate statistics (ie, Pearson correlation coefficients) were inspected. Then, exploratory factor analysis (EFA) using Principal Axis Factoring extraction and Varimax rotation was conducted for the KCES-R. Prior to interpreting findings from the EFA, the value of the KaiserMeyer-Olkin (KMO) value and Bartlett's Test of Sphericity were evaluated to ensure the data were factorable and to assess sampling adequacy. We planned to remove any items with low inter-item correlations $(<.3)$, and to place items with strong loadings on multiple factors $(>.4)$ in the factor with the best conceptual fit. The number of factors to retain was determined through joint examination of the scree plot and considering factors with eigenvalues $>1$. As confirmation, a parallel analysis Monte Carlo simulation was performed to create $95 \%$ confidence intervals of eigenvalues with 1000 parallel datasets from permutations of the raw data. ${ }^{9}$ Paired t-tests and Wilcoxon signed-rank tests were used to compare KCES-R domain scores of student pharmacists before and after a simulated patient encounter. KCES-R and KCES-PV empathy skill domain sum scores were compared using descriptive statistics.

Lastly, a thematic analysis of the student pharmacist reflections was also performed. Utilizing a grounded theory approach, a member of the research team (EW) reviewed the reflections and coded the responses into themes. The themes were checked by another member of the research team (AC) to ensure accuracy and to resolve any questions regarding the themes.

\section{RESULTS}

Of the 94 second-year student pharmacists who were invited to participate in the activity, all (100\%) participated in the activity, $94(100 \%)$ completed the pre-survey, and $90(96 \%)$ completed the post-survey. Thirty-six $(38 \%)$ of the student pharmacists were male and $70(74 \%)$ were white. Forty-five students were from CUSOP (48\%) and $49(52 \%)$ were from UW.

All items in the KCES-R had responses ranging from 1 to 7 . Item 5 had a skewness statistic of -2.5 and kurtosis statistic of 9.2, both outside of acceptable ranges. In addition, this item displayed a high mean (6.4). All other items in the KCES-R had skewness values between -2 and 2, and kurtosis values between -7 and 7; however, mean values for items were between 5.3 and 6.3, above the true midpoint of 4 . All items had significant $p$-values from the Shapiro-Wilk test, indicating non-normal distributions. However, due to the sample size of $>30$, and limited skewness and kurtosis issues, the data are reported using both parametric statistical tests for ease of presentation and interpretation, and nonparametric tests to ensure robustness of the results against possible normality assumption violations.

The EFA KMO value was .906 indicating 'marvelous' sampling adequacy, ${ }^{10}$ and Bartlett's Test of Sphericity was significant $(p<.001)$ indicating the data were factorable. Two factors were retained, global empathy beliefs (items 1-7) and personal empathy ability (items $8-14$ ), accounting for $74.2 \%$ of the variance in initial eigenvalues and $70.1 \%$ of the variance in rotation sums of squared loadings. Rotated factor loadings, inter-item correlations, and corrected item-scale correlations are shown in Table 1. Factor loadings for the global empathy beliefs domain ranged from .66-.90, and from $.75-.81$ for the personal empathy ability domain (Table 1). Within each domain, individual items had moderate to strong inter-item correlations, and corrected-item scale correlations ranging from .72-.88. Cronbach's alpha was .936 and .938 for the global and personal domains, respectively. Mean sum scores (Table 2) on each domain were well above the true midpoint of 28, and slight skewness $(-2.2)$ and kurtosis (7.8) were observed for the general domain. The two domains had a moderate Spearman's rank-order correlation of $.427(p<.001)$.

A comparison of the initial KCES-R administration (pre), and administration after a simulated patient encounter (post) is shown in Table 2 for the 90 individuals who completed both (linked using student generated codes). Significant changes in empathy domain scores were detected after the completion of a simulated patient experience $(p=.004$ and $p=$ .006 , respectively) in a paired t-test, with similar results for Wilcoxon Signed Rank Test. Individual items were also inspected; five items from each subscale were significantly higher after the experience (items 1, 2, 4, 6, 7, 8, 10, 11, 13, 14). The pre- and post-activity domain scores were also correlated (global views of empathy in health care $r=.659, p<$ .001 ; personal empathy ability $r=.669, p<.001)$.

SPs completed 93 (99\%) KCES-PV surveys after interacting with student pharmacists. A total of eight SPs rated 44 CUSOP students and seven SPs rated 49 UW students. In the present study, these observations were not linked to individual students, precluding a direct comparison of student evaluations of their own empathy ability with SP ratings. SP ratings of students, in regard to empathy ability, are presented and compared to student ratings in Table 3. Overall, it appears that SPs used a broader range of ratings of students than students did of themselves; students may have rated their own ability higher than SPs did. However, caution is advised in interpreting these numerical differences as student 
empathy ratings are nested by rater (with an average of 8 ratings per rater), and there was some small variation present across raters in terms of mean and dispersion of ratings.

Student pharmacists at CUSOP $(n=39)$ also completed reflections following the OSCE that they agreed to be used for research purposes. Table 4 displays the qualitative analysis of the student reflections. Key themes included students developing an understanding of what empathy is and the importance of practice in developing empathy and greater selfawareness. The utilization of practice along with feedback seemed to highlight areas for improvement, such as the impact of body language and listening skills.

\section{DISCUSSION}

Empathy is crucial for the development of a successful and effective pharmacist-patient relationship to build rapport with patients, increase trust and medication adherence, and improve patient health-related outcomes. ${ }^{1}$ This requires self-reflection of knowledge, skills, and ability to be empathetic. ${ }^{1}$ Empathy can be developed through experience, reflection, and feedback that is reinforced across multiple settings. ${ }^{1}$ This study provides validity evidence for use of the KCES-R in student pharmacists and introduces the KCES-PV for patient or SP use. Taken together, these two measures provide two methods of assessing students' skills in expressing empathy with patients (self-assessment and patient/SP rating).

Student pharmacists in the current project rated the importance of empathy very highly (global beliefs domain sum scores). ${ }^{11}$ Students' ratings regarding the importance of empathy may be attributed to an emphasis on empathy in their pharmacy school curricula. ${ }^{12,13}$ The literature would suggest that programs often utilize communication encounters, communication courses, OSCEs, and practice laboratories to address empathy. ${ }^{1}$ Thus, student pharmacists may perceive empathy as highly important due to the emphasis placed on it in their professional programs.

In the current project, students appeared to rate their empathy ability at a greater level than SP ratings of student empathy ability. There are several potential causes for this. First, students may overestimate their personal abilities. ${ }^{4}$ Second, there could be social desirability bias (overreporting of positive or more desirable attributes, such as growth in empathy, and minimizing negative or undesirable attributes) in student responses as well. Thus, it is highly likely that some students may rate their ability higher than is deserved after using the survey instrument or a simulated patient experience (often known as the Dunning Kruger effect). ${ }^{18}$ Lastly, it is interesting that global empathy beliefs and perceptions of one's own skills were moderately correlated; thus, perhaps the higher a student perceived the importance of empathy, the greater they rated their own ability, possibly linking again with social desirability bias.

Student pharmacist post-OSCE reflections revealed a conceptual understanding of empathy, but also a realization that practice was needed for further development as plans were made for subsequent encounters. This is an interesting finding since it may indicate that post-communication reflections for a specific interaction coupled with completion of a self-assessment of empathy may allow student pharmacists to more accurately assess their empathy skills and abilities. Deeper reflection may promote better self-awareness. For example, Smith, Norman, and Decety (2017) found multiple measures of student's empathy can allow for a more complete assessment. ${ }^{14}$ In our case, students' reflections of the specific interaction with the patient may produce a more vivid form of recall than mere completion of a self-assessment questionnaire of empathy. The specificity of the reflection questions may also have allowed the student pharmacists to better recollect their use of empathy for a specific health care interaction. Thus, use of patient ratings, watching the encounter, and then reflecting on the encounter may be a useful approach for students to grow and build self-awareness. Regular assessment and feedback are key to developing empathy in students. ${ }^{1}$ Using validated assessments that allow for self-evaluation and rater/evaluator/patient feedback can further contribute to the self-reflection process. Experiential learning and patient interactions, often in the clinical settings, can also contribute to the development of empathy. ${ }^{1}$ Future work could evaluate whether having student pharmacists re-assess themselves after watching a video of the encounter results in greater convergence of their responses with the patients' perceptions as well as how this develops during further patient interactions in experiential learning.

The KCES-R and KCES-PV are promising measures of empathy for health professional educators and researchers alike. The KCES has been used in thousands of student and health professional assessments of empathy internationally, including numerous articles in pharmacy education. Recently revised to reflect the validity evidence from those administrations, the KCES-R provides students with an opportunity to self-assess and report their perceived empathy skills. Self-assessment is an important skill for student pharmacists to develop as it aligns with CAPE domain 4.1. ${ }^{3}$ The KCES-PV affords the patient the opportunity to provide their perspective of the student pharmacists' ability to show empathy. Obtaining the patient's perspective is essential for more comprehensive insight into the student's demonstration of empathy. This external feedback provides a more complete and personally unbiased perspective of the student pharmacist's true ability to empathize with patients and can be useful for the identification of specific aspects of student 
empathy in need of further development. ${ }^{15,16}$ This study focused on the KCES-PV use for rating student empathy ability, but future study in other settings (ie, health care provider empathy in primary care) could expand the use of this scale to aid clinician professional development by providing a method of obtaining patient feedback.

The limitations of this study include the use of two institutions for implementation. Therefore, results may not be generalizable to other institutions; the student population that participated in this project may not be a representative sample of other student pharmacists. National statistics indicate that $37 \%$ of PharmD graduates are male; our sample was a little lower at 30\%. Fall 2020 student enrollments indicated that approximately $48 \%$ of students were white; our sample was higher at $70 \% .{ }^{17}$ Moreover, this study used SPs patient care experiences, and therefore, may not be illustrative of patient care that is provided to patients in actual practices. Due to the nature of this study, student and SP responses could not be linked which limited the ability to compare responses, and demonstration of validity evidence for the KCES-PV was limited. Only one patient case scenario was utilized for this study; thus, case specificity effect could have occurred. This case presented a homeless patient at a community pharmacy picking up a new prescription (Levetiracetam) following an ER visit for a seizure. Due to the somewhat complex nature of the patient scenario, student pharmacists may have either missed or not recognized opportunities to show empathy. The complexity of the patient scenario also may be a strength of this study, as from this single patient encounter alone student ratings of empathy beliefs and skills increased. Lastly, as mentioned above, the responses of study participants could be subject to social desirability bias.

\section{CONCLUSION}

Student pharmacists must develop self-awareness of how they are perceived and should practice demonstrating empathy, an important skill, to patients. Intentional use of an instrument to consider one's own empathy beliefs and abilities (KCES-R), a tool to capture a patient's views of those abilities (KCES-PV), and a process for reflection may help grow student pharmacist self-awareness and empathy. The KCES-R and KCES-PV are promising measures of empathy for health professional educators and researchers. Utilization of the KCES-R and the KCES-PV provides a more comprehensive assessment of a student pharmacist's ability to empathize with patients, which can assist in the identification of specific aspects of student empathy in need of further development.

\section{ACKNOWLEDGMENT}

The authors report no conflicts of interest. Support for this project was funded by an Internal Grant from Cedarville University School of Pharmacy.

\section{REFERENCES}

1. Ratka A. Empathy and the development of affective skills. Am J Pharm Educ 2018;82(10):7192. doi:10.5688/ajpe7192

2. Accreditation Council for Pharmacy Education. Accreditation standards and key elements for the professional program in pharmacy leading to the Doctor of Pharmacy degree: "Standards 2016". Accessed June 30, 2021, https://www.acpe-accredit.org/pdf/Standards2016FINAL.pdf

3. Medina MS, Plaza CM, Stowe CD, et al. Center for the Advancement of Pharmacy Education 2013 educational outcomes. Am J Pharm Educ 2013;77(8):162.

4. Karpen SC. The social psychology of biased self-assessment. Am J Pharm Educ 2018;82(5):6299-6299. doi: 10.5688 /ajpe6299.

5. Sturpe DA. Objective structured clinical examinations in doctor of pharmacy programs in the United States. $A m J$ Pharm Educ 2010;74(8):148. doi: 10.5688/aj7408148.

6. Tatachar A, Wettergreen S, Summers S. Reflective metacognition and objective structured clinical examination performance in introductory pharmacy practice experiences. Curr Pharm Teach Learn 2020;12(10):1208-1214. doi: https://doi.org/10.1016/i.cptl.2020.04.030.

7. Kiersma ME, Chen A, Yehle KS, Plake KS. Validation of an empathy scale in pharmacy and nursing students. Am J Pharm Educ. 2013;77(5):94. doi: 10.5688/ajpe77594

8. Aronson B, Chen AMH, Blakely ML, Kiersma ME, Wicker E. An update on the Kiersma Chen Empathy Scale (KCES): creating the KCES-Revised. Am J Pharm Educ (under review).

9. O'Connor BP. SPSS and SAS programs for determining the number of components using parallel analysis and velicer's MAP test. Behav Res Methods Instrum Comput 2000;32(3):396-402. doi: 10.3758/bf03200807.

10. Kaiser HF. An index of factorial simplicity. Psychometrika 1974;39(1):31-36. doi: 10.1007/BF02291575.

11. Bleakley A, Bligh J. Students learning from patients: let's get real in medical education. Adv Health Sci Educ Theory Pract 2008;13(1):89-107. doi: 10.1007/s10459-006-9028-0. 
12. Jubraj B, Barnett NL, Grimes L, Varia S, Chater A, Auyeung V. Why we should understand the patient experience: clinical empathy and medicines optimisation. Int J Pharm Pract 2016;24(5):367-70. doi: 10.1111/ijpp.12268.

13. Tamayo CA, Rizkalla MN, Henderson KK. Cognitive, behavioral and emotional empathy in pharmacy students: targeting programs for curriculum modification. Front Pharmacol 2016;7:96. doi: 10.3389/fphar.2016.00096.

14. Smith KE, Norman GJ, Decety J. The complexity of empathy during medical school training: evidence for positive changes. Med Educ 2017;51(11):1146-1159. doi: 10.1111/medu.13398.

15. Watanabe S, Yoshida T, Kono T, et al. Relationship of trainee dentists' self-reported empathy and communication behaviors with simulated patients' assessment in medical interviews. PLoS One 2018;13(12):e0203970. doi: 10.1371/journal.pone.0203970.

16. Bernardo MO, Cecilio-Fernandes D, Lima ARdA, et al. Investigating the relation between self-assessment and patients' assessments of physicians-in-training empathy: a multicentric, observational, cross-sectional study in three teaching hospitals in Brazil. BMJ Open 2019;9(6):e029356-e029356. doi: 10.1136/bmjopen-2019-029356.

17. American Association of Colleges of Pharmacy. Student applications, enrollments and degrees conferred reports. Accessed October 11, 2021, https://www.aacp.org/research/institutional-research/student-applicationsenrollments-and-degrees-conferred

18. Psychology Today. Dunning-Kruger effect. Accessed October 12, 2021, https://www.psychologytoday.com/us/basics/dunning-kruger-effect

Figure 1. Overview of Methods

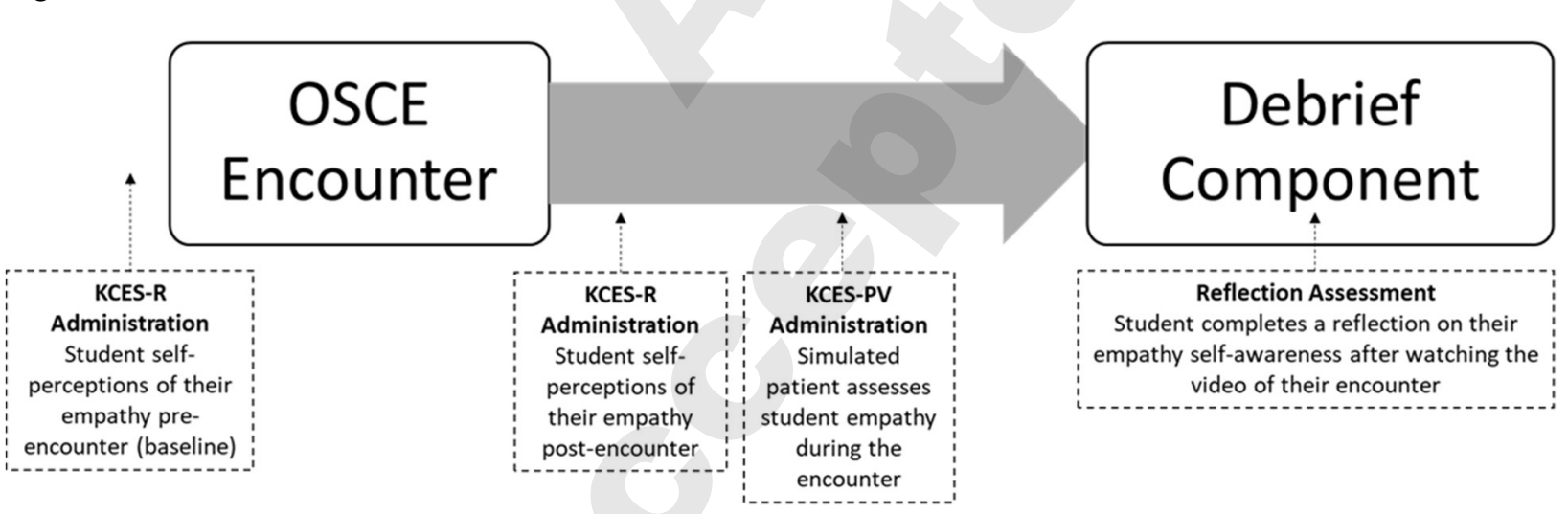

OSCE $=$ Objective Structured Clinical Examination 
Table 1. KCES-R Factor Loadings, Inter-Item Correlations, and Corrected Inter-Item Correlations

\begin{tabular}{|c|c|c|c|c|}
\hline Item & Factor 1 & Factor 2 & IIC $^{\mathrm{a}}$ & $\mathrm{ITC}^{\mathrm{b}}$ \\
\hline
\end{tabular}

Global Empathy Beliefs

$\begin{array}{llll}\text { 1. Comprehend patient experiences } & .71 & .55-.73 & .72\end{array}$

2. Express an understanding of patients' feelings $\quad \begin{array}{lll}.87 & .59-.89 & .87\end{array}$

3. Value patients' point of view $\quad \begin{array}{lll}90 & .64-.89 & .88\end{array}$

$\begin{array}{lllll}\text { 4. Consider patients' feelings to provide patient-centered care } & .81 & .61-.82 & .82\end{array}$

5. Be caring in order to build a strong relationship with patients $\quad .87 \quad 66-.83 \quad .87$

6. Identify with patients' feelings $\quad .73 \quad$ 55-.76 $\quad .75$

7. View the world from patients' perspective $\quad .66 \quad .56-.66 \quad .72$

Personal Empathy Ability

8. Comprehend patient experiences $\quad \begin{array}{lll}.80 & .62-.76 & .77\end{array}$

$\begin{array}{lllll}\text { 9. Express an understanding of patients' feelings } & .81 & .63-.79 & .81\end{array}$

$\begin{array}{llll}\text { 10. Value patients' point of view } & .77 & .63-.80 & .81\end{array}$

11. Consider patients' feelings to provide patient-centered care $\quad \begin{array}{lll}.80 & .64-.80 & .84\end{array}$

12. Be caring in order to build a strong relationship with patients $\quad \begin{array}{lll}.76 & .62-.79 & .80\end{array}$

13. Identify with patients' feelings $\quad \begin{array}{llll}.78 & .65-.79 & .81\end{array}$

$\begin{array}{llll}\text { 14. View the world from patients' perspective } & .75 & .60-.73 & .75\end{array}$

KCES-R Kiersma Chen Empathy Scale Revised version; ${ }^{a}$ inter-item correlations among items within factor; ${ }^{\mathrm{b}}$ corrected itemscale correlation for items within factor 


\begin{tabular}{|c|c|c|c|}
\hline Item & $\begin{array}{l}\text { Pre-Survey } \mathrm{N}=94 \\
\text { Mean (SD) }\end{array}$ & $\begin{array}{l}\text { Post-Survey } \mathrm{N}=90 \\
\text { Mean (SD) }\end{array}$ & $p$ value \\
\hline \multicolumn{4}{|l|}{$\begin{array}{l}\text { Global Views. How necessary is it for healthcare } \\
\text { professionals to be able to... }\end{array}$} \\
\hline Comprehend patient experiences & $6.20(1.12)$ & $6.44(0.80)$ & \\
\hline Express an understanding of patients' feelings & $6.21(1.00)$ & $6.44(0.72)$ & \\
\hline Value patients' point of view & $6.32(1.04)$ & $6.47(0.78)$ & \\
\hline $\begin{array}{l}\text { Consider patients' feelings to provide patient-centered } \\
\text { care }\end{array}$ & $6.18(1.07)$ & $6.49(0.76)$ & \\
\hline $\begin{array}{l}\text { Be caring in order to build a strong relationship with } \\
\text { patients }\end{array}$ & $6.39(1.00)$ & $6.53(0.74)$ & \\
\hline Identify with patients' feelings & $5.99(1.37)$ & $6.34(0.86)$ & \\
\hline View the world from patients' perspective & $5.90(1.36)$ & $6.34(0.85)$ & \\
\hline Sum of Global Empathy Views domain & $43.2(6.82)$ & $44.9(4.79)$ & $.004 *$ \\
\hline \multicolumn{4}{|l|}{ Personal Ability. I am able to... } \\
\hline Comprehend patient experiences & $5.44(1.08)$ & $5.67(1.07)$ & \\
\hline Express an understanding of patients' feelings & $5.59(1.18)$ & $5.62(1.19)$ & \\
\hline Value patients' point of view & $5.95(1.11)$ & $6.19(0.97)$ & \\
\hline $\begin{array}{l}\text { Consider patients' feelings to provide patient-centered } \\
\text { care }\end{array}$ & $5.78(1.07)$ & $6.07(1.06)$ & \\
\hline $\begin{array}{l}\text { Be caring in order to build a strong relationship with } \\
\text { patients }\end{array}$ & $6.01(1.07)$ & $6.16(1.08)$ & \\
\hline Identify with patients' feelings & $5.67(1.15)$ & $5.81(1.23)$ & \\
\hline View the world from patients' perspective & $5.34(1.15)$ & $5.81(1.22)$ & \\
\hline Sum of Personal Empathy Ability domain & $39.8(6.68)$ & $41.3(6.58)$ & $.006 *$ \\
\hline
\end{tabular}

KCES-R Kiersma Chen Empathy Scale Revised version

+Items had response categories ranging from 1 to 7 with anchors at 1 'Unnecessary', 4 'Moderately necessary', and 7

'Extremely necessary'

^Items had response categories ranging from 1 to 7 with anchors at 1 'Does not describe me', 4 'Describes me moderately well', and 7 'Describes me extremely well'

*Paired samples T-test was used to determine significance, defined as $\mathrm{p}<.05$ 
Table 3. Comparison of Post-Activity KCES-R and KCES-PV Ability Domain

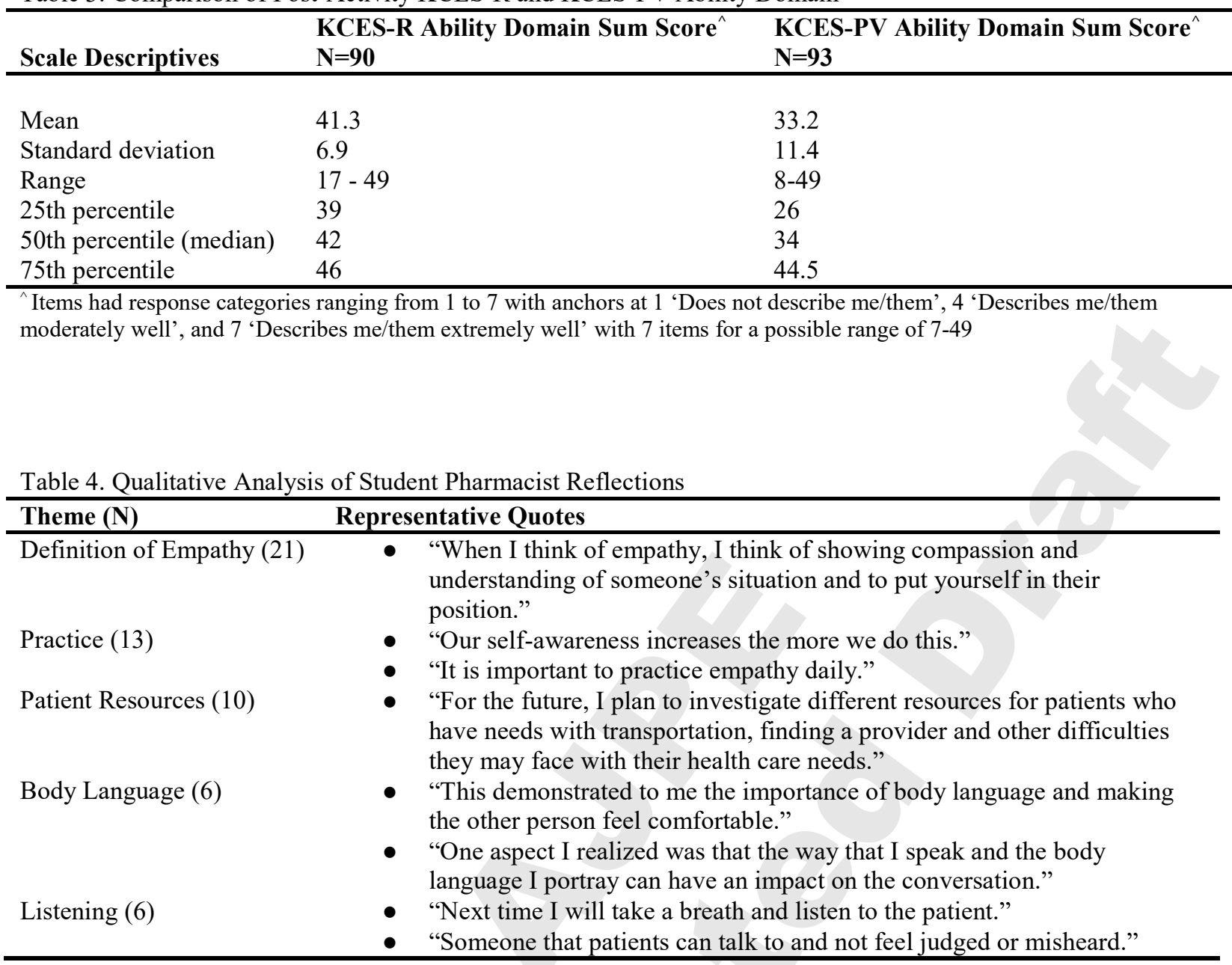

